\title{
Diabetic Ketoacidosis in a Patient With COVID-19
}

Anthony Stack, DO, MS • Lauren Terpak, MD • Ghania Masri, MD

A 40-year-old man with a history of type 1 diabetes and dyslipidemia presented to the emergency department with a 5-day history of polydipsia, polyuria, and feelings of dehydration. The patient also reported a 3-week history of a resolving unproductive cough without dyspnea or any further respiratory or infectious symptoms. Although the patient's hemoglobin $\mathrm{A}_{1 \mathrm{c}}$ level was $11.5 \%$, he reported adherence to his home regimen of insulin 70/30.

At presentation, he was afebrile and had a respiratory rate of 23 breaths/min and an oxygen saturation of $95 \%$ on ambient air. He had signs of dehydration on examination; however, lung and abdominal examination findings were unremarkable.

Laboratory tests revealed a serum glucose level of $328 \mathrm{mg} / \mathrm{dL}$, a high anion gap of $20 \mathrm{mEq} / \mathrm{L}$, a decreased bicarbonate level of $18 \mathrm{mEq} / \mathrm{L}$, and an elevated $\beta$-hydroxybutyrate level of 43.3 $\mathrm{mmol} / \mathrm{L}$. His lactate level was within the normal range, and other causes of ketoacidosis were ruled out.

The patient was diagnosed with diabetic ketoacidosis (DKA) and was started on intravenous fluids and insulin. An infectious workup was initiated to identify a possible precipitating etiology. The procalcitonin level was $0.30 \mathrm{ng} / \mathrm{mL}(<0.50 \mathrm{ng} / \mathrm{mL}$ indicates that systemic infection is less likely), results of a standard respiratory viral panel were negative, urinalysis findings were unremarkable, and urine Streptococcus pneumoniae and Legionella antigen test results were negative. However, a chest radio-

\section{AFFILIATIONS:}

University of Florida College of Medicine, Jacksonville, Florida

\section{CITATION:}

Stack A, Terpak L, Masri G. Diabetic ketoacidosis in a patient with COVID-19. Consultant. 2020;60(9):3-4. doi:10.25270/con.2020.06.00021

Received May 6, 2020. Accepted May 21, 2020.

\section{DISCLOSURES:}

The authors report no relevant financial relationships.

\section{CORRESPONDENCE:}

Anthony Stack, DO, MS, University of Florida College of Medicine, 4th Floor, Learning Resources Center, 653-1 W 8th St, L18, Jacksonville, FL 32209 (anthony.stack@jax.ufl.edu) graph revealed patchy bibasilar opacifications (Figure).

Based on the radiographic findings and the overall clinical picture, a nasopharyngeal swab using reverse transcription polymerase chain reaction testing (with a Roche Cobas analyzer) was obtained and returned positive for severe acute respiratory syndrome coronavirus 2 (SARS-CoV-2), the virus responsible for COVID-19.

The patient was continued on intravenous fluids and insulin until resolution of DKA. He was then resumed on his home subcutaneous insulin regimen and demonstrated appropriate glycemic control. He was monitored closely for signs of worsening COVID-19 symptoms and received supportive care. His cough improved, and he was discharged home with strict instructions to self-isolate for at least 14 days

Discussion. DKA is a serious acute complication of diabetes, characterized by hyperglycemia and ketoacidosis. DKA typically evolves very rapidly, with patients presenting with hyperglycemia, mental status changes, and abdominal pain. A precipitating event usually is identified; infection, insulin nonadherence, or increased insulin requirement are the most common trigger events. ${ }^{1}$ In addition to providing intravenous fluids and insulin and correcting other electrolyte abnormalities, treating the underlying insult is an essential part of DKA management.

Infection and other forms of acute illness are thought to precipitate DKA by provoking an inflammatory state that induces the release of counterregulatory hormones such as glucagon, cortisol, catecholamines, and growth hormone, which leads to hyperglycemia and ketosis. ${ }^{1}$ Furthermore, recent research has suggested that, in addition to provoking an acute inflammatory state, the SARS-CoV-2 virus also may damage the pancreatic islet cells, further contributing to hyperglycemia and inducing ketosis. ${ }^{2}$ COVID-19 may also accelerate fat breakdown and induce ketosis. ${ }^{7}$ Anecdotal reports have shown worsening glycemic control with increasing insulin requirements in patients with COVID-19. ${ }^{3}$

The findings in this patient's case suggest that COVID-19 may have precipitated DKA. While the patient presented with symptoms of hyperglycemia and cough, chest radiography findings were concerning for COVID-19. Common abnormal 


\section{Case in Point}

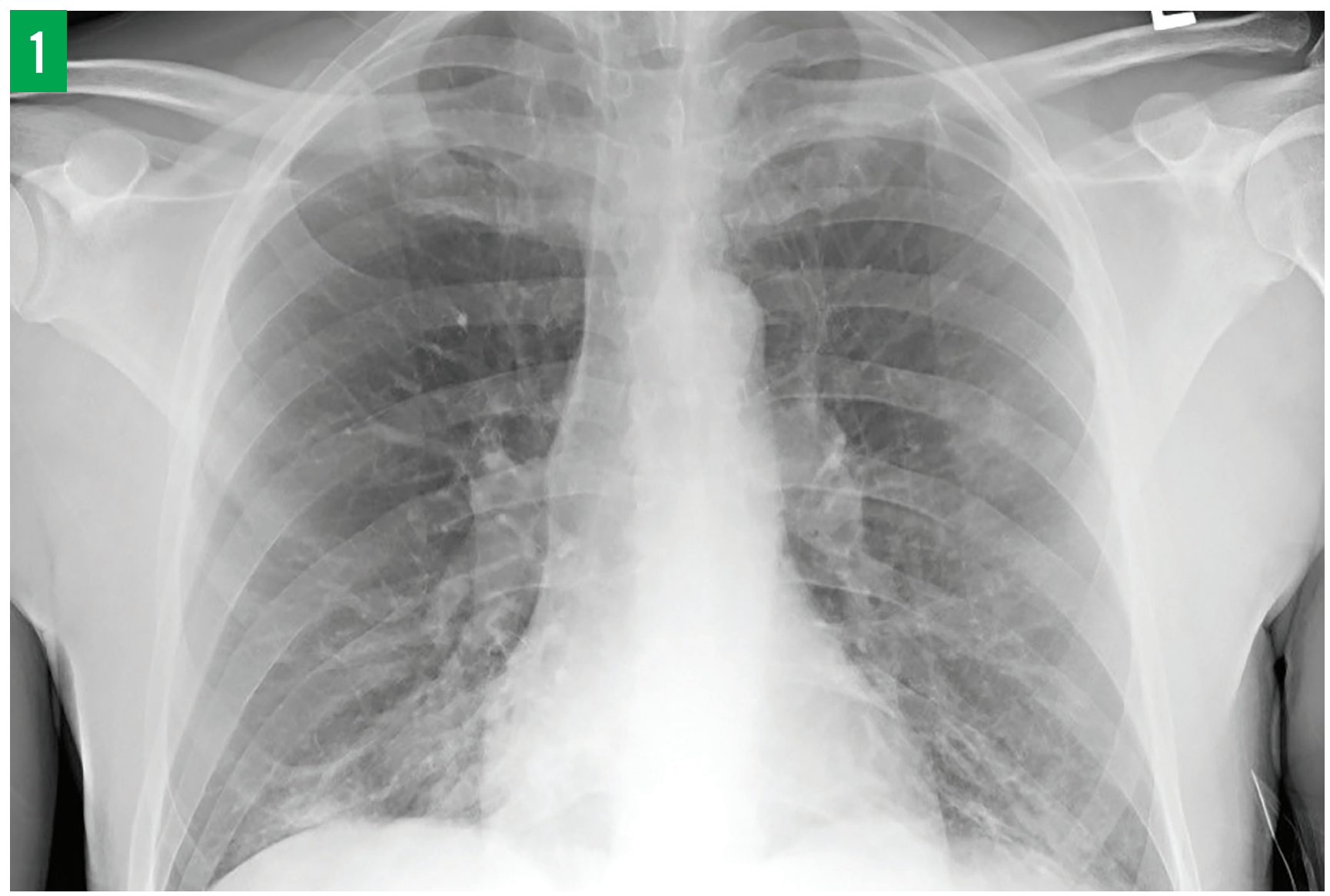

The patient's chest radiograph showing patchy bibasilar opacifications.

radiographic features in a COVID-19-positive patient include consolidation or ground-glass opacities with bilateral, peripheral, and lower lung field distribution. These findings may be less noticeable or absent in early or mild disease but tend to increase over the course of the illness. ${ }^{4}$

Although persons with diabetes do not have an increased risk of contracting COVID-19, they may have a higher chance of experiencing serious complications from the virus. Furthermore, according to a recent Chinese case series, the severity of COVID-19 in persons with diabetes may be prematurely obscured by an initial milder presentation of the infection. ${ }^{2}$ It is prudent to not underestimate the severity of COVID-19 in patients with diabetes who present without the classic symptoms of the virus. Results of a recent Chinese study involving 658 hospitalized patients with confirmed COVID-19 suggest that the virus may induce ketoacidosis, leading to increased length of hospital stay and mortality. ${ }^{7}$

Individualized blood glucose target goals and treatment strategies should be made according to specific circumstances of COVID-19 in patients with diabetes. ${ }^{5}$ The need for a simultaneous patient-centered approach in routine diabetes care has to be coordinated by a multidisciplinary team amid the ongoing

\section{COVID-19 pandemic. $^{6}$}

This case highlights the importance of considering COVID-19 infection in a patient presenting with signs and symptoms of DKA. It also provides evidentiary support of reports suggesting that patients with diabetes may have increased risk of serious adverse events when infected with the COVID-19 virus.

\section{REFERENCES:}

1. Kitabchi AE, Umpierrez GE, Miles JM, Fisher JN. Hyperglycemic crises in adult patients with diabetes. Diabetes Care. 2009;32(7):1335-1343. doi:10.2337/dc09-9032

2. Guo W, Li M, Dong Y, et al. Diabetes is a risk factor for the progression and prognosis of COVID-19. Diabetes Metab Res Rev. 2020:e3319. doi:10.1002/ dmrr.3319

3. Tucker ME COVID-19 and diabetes: known mechanisms and a 'new beast'? Medscape. April 14, 2020. Accessed May 21, 2020. https://www.medscape. $\mathrm{com} /$ viewarticle/928629

4. Wong HYF, Lam HYS, Fong AH-T, et al. Frequency and distribution of chest radiographic findings in COVID-19 positive patients. Radiology. 2020; 201160. doi:10.1148/radiol.2020201160

5. Ma W-X, Ran X-W. The management of blood glucose should be emphasized in the treatment of COVID-19 [in Chinese]. Sichuan Da Xue Xue Bao Yi Xue Ban. 2020;51(2):146-150. doi:10.12182/20200360606

6. Banerjee M, Chakraborty S, Pal R. Diabetes self-management amid COVID 19 pandemic. Diabetes Metab Syndr. 2020;14(4):351-354. doi:10.1016/j. dsx.2020.04.013

7. Li J, Wang X, Chen J, Zuo X, Zhang H, Deng A. COVID-19 infection may cause ketosis and ketoacidosis. Diabetes Obes Metab. Published online April 20, 2020. doi:10.1111/dom.14057 